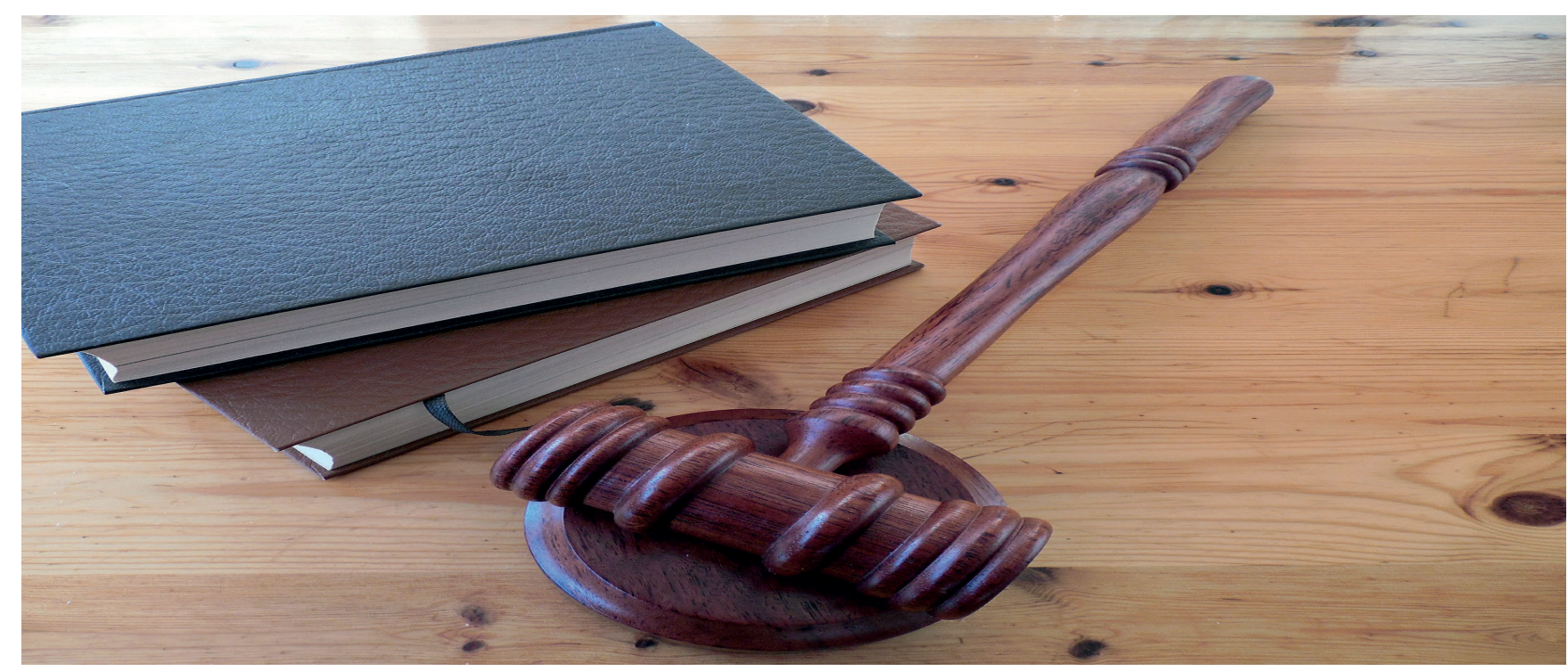

Tomado de: https://pixabay.com/es/photos/martillo-libros-ley-tribunal-620011/

\title{
SARS-CoV2 y sus efectos en la aplicación del derecho de la competencia
}

\section{SARS-CoV2 and its effects on the application of competition law}

Celso Pérez-Amaro*

\section{Resumen}

La competencia económica ha sido un factor importante en el desarrollo de los mercados en el mundo, las autoridades en esta materia se han caracterizado en los últimos años por endurecer su postura frente a las prácticas colusivas o anticompetitivas, sin embargo, la aparición del SARS-Cov2 ha puesto en entredicho las prioridades de la política antitrust en el ámbito internacional; en este trabajo se expone la diversidad de posturas de las autoridades de competencia y la redefinición de los lineamientos y las excepciones a la ley motivadas por la pandemia actual.

Palabras clave: SARS-CoV2, competencia económica, prácticas monopólicas.

\begin{abstract}
Economic competition has been an important factor in the development of markets in the world. In the last years, experts in this field had hardened their stance against collusive or anti-competitive practices. However, the appearance of SARS- Cov2 has questioned the antitrust policy priorities in the international context. This study analyses the diversity of positions from competition authorities and the redefinition of regulations and exceptions to the law as a result of the current pandemic.
\end{abstract}

Keywords: SARS-CoV2, competencia económica, prácticas monopólicas.

*Fecha de recepción: 06/11/2020 Fecha de aceptación: 30/11/2020 Correspondencia: ceperez@docentes.uat.edu. mx Dirección: Facultad de Derecho y Ciencias Sociales Victoria. Universidad Autónoma de Tamaulipas. 


\section{INTRODUCCIÓN}

La defensa de la competencia (libre competencia, libre concurrencia, competencia económica, monopolios, prácticas monopólicas y concentraciones), se encuentra contenida en el artículo 28 de la Constitución Política de los Estados Unidos Mexicanos, dicha base se remonta a la Constitución de 1857, aunque muy elemental en su tiempo fue innovadora, lamentablemente se tuvo una ley reglamentaria efectiva hasta el año de 1992, con la Ley Federal de Competencia Económica.

Actualmente se cuenta con la Ley Federal de Competencia Económica publicada en el Diario Oficial de la Federación (DOF), con fecha 23 de mayo de 2014, que abrogó la del 24 de diciembre de 1992. La Comisión Federal de Competencia Económica es el órgano autónomo que tiene por objeto garantizar la libre concurrencia y competencia económica, así como investigar y en su caso sancionar todas las prácticas que atenten contra ello.

A partir de la llegada del SARS-CoVid2 en el año 2019, el mundo ha tenido que adaptarse a una nueva normalidad, desde la forma en que se educa, hasta la manera de realizar operaciones mercantiles o comerciales. Al mismo tiempo, los gobiernos pretenden proteger a la población del contagio de la enfermedad estableciendo restricciones, tal es el caso de muchas actividades que fueron restringidas, situación que provocó el cierre de algunas pequeñas y medianas empresas (pymes), (Secretaría de Salud, 2020).

Los alcances de impacto económico no se observan solamente en un sector en lo particular, más bien afecta al grueso de la economía (Baldwin, R., Weder Di Mauro, B. 2020). Como resultado de lo anterior, las empresas o agentes económicos, se han visto en la necesidad de buscar alternativas para sobrevivir ante esta contingencia. En el presente estudio, se expone el efecto que ha tenido la pandemia en la toma de decisiones de las autoridades de defensa de la competencia en algunos países. Los efectos de la pandemia ocasionada por el
SARS-CoVid2, han despertado el interés por el estudio del comportamiento de las autoridades antitrust, por el momento se han realizado estudios sobre ello (Baldwin, R., Weder Di Mauro, B. 2020; Irrazabal, 2020; Alemano, 2020; Thomas, 2020; Costa-Cabral, 2020; Petros, 2020), entre otros; debido a la cercanía de las circunstancias que la provocaron no se identifican publicaciones de libros, sin embargo, existen estudios en artículos que exploran el tema, así como las declaraciones y comunicados de las autoridades.

El presente trabajo servirá de base para conocer el desarrollo de la flexibilidad o inflexibilidad de las autoridades antitrust y, los efectos que sus decisiones han tenido en la economía.

\section{La competencia económica}

El sistema económico que permite la participación del capital privado como fuente o motor del desarrollo económico de los países, también llamada economía de mercado, busca que las sociedades puedan alcanzar más bienestar social a través de la participación económica de los ciudadanos, sin embargo, la experiencia y la historia han mostrado que para que ese supuesto se presente, al menos de forma más visible, es necesario que se den los elementos que se requieren para su funcionamiento (Pérez, 2008).

Hasta el siglo XVIII todos los actos que realizaban los consumidores y las empresas se consideraban como un proceso de suma cero, es decir las ganancias de una persona son la pérdida de otra, esto fortaleció en su momento el sistema mercantilista.

A pesar de que el mercantilismo tuvo un auge importante en el mismo siglo XVIII, inició el análisis y determinación sobre las transacciones comerciales que traía como consecuencia beneficios a las partes, y que se podían alcanzar mejores resultados si se alejaba un poco el control rígido del Estado. Adam Smith quién para muchos es considerado el padre de la economía, acuñó en su libro La Riqueza de las Naciones el término de la mano invisible, que indicaba que se podía obtener o lograr el interés 
público mediante la búsqueda de un interés propio o particular por parte de las empresas (Samuelson, 1999).

Existen mercados en donde se presenta la competencia imperfecta, se les considera en ocasiones como mercados imperfectamente competitivos (Samuelson, 1999), debido a que los costes, las barreras a la entrada y la interacción estratégica inciden en ello.

Por un lado, la competencia económica puede beneficiar y, por otro lado, puede perjudicar, normalmente beneficia a los consumidores y a las empresas que buscan una participación en los mercados, sin embargo, en sentido contrario, hay agentes económicos que se ven perjudicados al momento en que pierden los beneficios de monopolio o bien, de poder económico.

Dentro de un análisis de los mercados, la competencia provoca usualmente la disminución de precios en los productos, casi hasta el nivel de los costos de producción, incluidas las ganancias respectivas, por lo que si uno de los competidores decide subir los precios de su producto o servicio, los consumidores reflejarían sus preferencias hacia el resto de los ofertadores, esta consideración es aceptable, sin embargo, debe existir un respeto y cumplimiento a las disposiciones o normas del derecho de la competencia económica para que los supuestos se puedan dar. Por ejemplo, la situación de que exista una libre concurrencia a los mercados, no garantiza que los agentes económicos no van a cometer acuerdos colusivos o prácticas anticompetitivas entre ellos, con el fin de obtener mayores ganancias (Pérez, 2008).

Como se ha visto, el proceso de libre competencia y libre concurrencia en los mercados debe acompañarse con una legislación, que invite y conmine a los agentes económicos a respetar los supuestos establecidos en la ley. De ahí que sea relevante la legislación que garantice la competencia, en todos los países que cuentan con una economía de mercado.
El derecho de la competencia económica que aparece de manera importante en los Estados Unidos de América, mediante un proyecto que se presentó al Congreso, a través del cual el Senador John Sherman provocó la aprobación y promulgación de la Ley Sherman, en el mes de julio del año de 1890, es considerada la primer ley antimonopolio en el mundo.

Se ha mencionado que el motivo de esta ley tiene su origen en situaciones de tipo político más que de razones económicas (Fernandez, 2000), otros, por el contrario, consideran que la promulgación de la ley tuvo que ver con problemas que se tenían por la comisión de actos abusivos de algunos dirigentes de las industrias, mismos que se denominaron robber barons y se presentaron principalmente en los sectores del transporte ferroviario y del acero (Pereznieto, 2002).

De alguna manera, la entrada en vigor de la ley Sherman trajo consigo el debilitamiento del poder económico en manos de unos pocos, así como la protección del mercado, es decir, la mejora en la libre concurrencia y la libre competencia, además, se frenaron las conductas predatorias de precios.

Uno de los primeros casos que llamó la atención fue el de Microsoft (Becker, 1998), que se originó por la dificultad de establecer si Internet Explorer es un producto separado o forma parte de un sistema operativo integrado (Shapiro, 1999).

Entonces, el derecho de la competencia económica ha servido para vigilar el comportamiento que interfiere en el buen desempeño de los mercados y de la libre competencia (Cooter y Ulen, 1998).

En ese sentido la tarea de velar, vigilar y sancionar es propia del Estado, de alguna manera interviene inicialmente con la elaboración de la legislación antimonopolio, que fija las bases del comportamiento de los agentes económicos (Pascual y Vicente, 2000). 
Actualmente, con autoridades fortalecidas en todos los países de la Organización para la Cooperación y el Desarrollo Económicos (OC$\mathrm{DE})$, y con leyes antimonopolio en todos ellos, se sigue en la lucha de vigilar y sancionar todas aquéllas conductas que dañen la libre competencia, la libre concurrencia y, en consecuencia, a los consumidores de bienes y servicios.

Ahora se analizarán las medidas que se han implementado en algunos países, buscando sancionar las prácticas anticompetitivas y, por otro lado, permitir los acuerdos entre agentes económicos, mismos que normalmente son prohibidos, pero en razón de las necesidades generadas por el SARS-CoV2, se han puesto a discusión.

\section{SARS-CoV2 y comportamiento de vigilancia de las autoridades antimonopolio}

La defensa de la competencia ha sido un tema importante para el desarrollo económico de los países, especialmente a partir de la pandemia mundial provocada por el virus denominado SARS-Cov2. En ese sentido, existen comportamientos o acuerdos de colaboración que se pueden llevar a cabo entre agentes económicos para salir adelante frente a una crisis de esta naturaleza, es importante analizar hasta qué punto las autoridades de defensa de la competencia permitirán acuerdos o colusiones entre los diferentes actores de la economía para poder salir adelante.

Con el avance de la pandemia, se observa con atención el comportamiento de la economía, en esta nueva realidad, y las autoridades de defensa de la competencia de todos los países buscan, como ya se dijo, entender a los agentes económicos, pero por otro lado, vigilan su comportamiento para evitar que dichos agentes económicos aprovechen el relajamiento de las disposiciones antitrust, para obtener beneficios fuera del marco legal.

Las consecuencias económicas generadas a raíz de la pandemia han sido diversas, por un lado, tenemos que algunas autoridades de de- fensa de la competencia se han vuelto más agresivas en el cumplimiento de las reglas antimonopólicas y, por otro lado, hay otras autoridades de defensa de la competencia, que han relajado el cumplimiento dela legislación antitrust, considerando que para las empresas o agentes económicos no es sencillo afrontar la falta de consumo provocado por el COVID19.

El presente trabajo tiene por objeto conocer el comportamiento de las autoridades de defensa de la competencia, en diversos países, durante la llamada nueva normalidad. A continuación, se expondrán las acciones o medidas que las autoridades antitrust han realizado, entre ellas, el inicio de investigaciones en sectores sensibles para los consumidores.

\section{Medidas e investigaciones para evitar prácti- cas anticompetitivas}

Una de las preocupaciones más importantes de las autoridades antitrust en el mundo, es la comisión de prácticas anticompetitivas, por parte de los agentes económicos, sobre todo, en sectores sensibles relacionados con la pandemia. En seguida se citarán algunas medidas que han establecido diversos países, para evitar el perjuicio de los consumidores como consecuencia de las prácticas anticompetitivas.

En México, la Comisión Federal de Competencia Económica, a través del Pleno, decidió multar con 626 millones 427 mil pesos, a 11 empresas y 14 personas físicas que se coludieron para la presentación o abstención de posturas en diversas licitaciones en perjuicio del erario y de los derechohabientes, dichas prácticas generaron un daño al erario y a los derechohabientes de al menos mil doscientos millones de pesos (COFECE-031-2020).

En el expediente IO-001-2020, la COFECE investigó las posibles prácticas monopólicas relativas, en el mercado de la producción, distribución y comercialización de oxígeno me- 
dicinal en el país. El inicio de la investigación fue por la posible comisión de prácticas monopólicas en el establecimiento de condiciones de exclusividad, en la compra o venta del mercado de producción, distribución y comercialización de oxígeno medicinal y servicios relacionados en el territorio nacional.

La Comisión aclaró que el inicio de esta investigación, fue una acción para verificar el cumplimiento de la Ley Federal de Competencia Económica, como medida preventiva, ya que el oxígeno, objeto del estudio, se utiliza como parte de los tratamientos de atención de pacientes con problemas respiratorios, entre ellos, pacientes con padecimientos provocados por el SARS-CoV2. Dentro de la investigación se incluyeron los servicios a domicilio de ventilación mecánica o invasiva, así como la no invasiva, además del empleo en intervenciones quirúrgicas, situaciones que en este tiempo de pandemia, deben de seguirse muy de cerca por las implicaciones directas que tienen en la salud de la población (COFECE-030-2020).

Por su parte, la Competition Bureau of Canada, en su declaración del 20 de marzo de 2020, a través de su Comisionado Matthew Boswell, se pronunció respecto de la aplicación de las leyes antimonopolio durante el SARS-CoVid2, enviando un mensaje a los canadienses de que la Comisión se mantendrá atenta y alerta sobre las prácticas o conductas anticompetitivas por parte de las empresas que, de manera deshonesta, buscan beneficiarse y aprovecharse de los consumidores y empresas durante este periodo de pandemia.

La Comisión canadiense pone énfasis en las prácticas de marketing engañosas, así como en las prácticas de colusión entre competidores. Sin embargo, asegura que las leyes y autoridades de competencia económica se adaptan a los acuerdos de colaboración entre empresas, que sean favorables para la oferta asequible de bienes y servicios, y que sean benéficos para los consumidores (Competition $\mathrm{Bu}-$ reau Canada, 2020).
El otro integrante del T-MEC, Estados Unidos, a través del Departamento de Justicia, advierte a los empresarios sobre la violación de las leyes antimonopolio en la fabricación, distribución y venta de productos de salud pública. El 9 de marzo de 2020, este país emite esta declaración para dejar en claro que, sus autoridades antimonopolio investigarán y sancionarán a las personas o empresas que violen las leyes antimonopolio, sobre todo en la fabricación, distribución o venta de mascarillas, respiradores y diagnósticos.

Esta declaración busca que las empresas no aprovechen este momento de contingencia sanitaria para aumentar ganancias con el incremento de precios, o bien, con la colusión o acuerdos entre las mismas. Pero la autoridad norteamericana va más allá, ya que subraya que las personas o empresas que fijen precios, o manipulen la oferta de equipos de protección de la salud, como guantes esterilizados y máscaras faciales, además de las sanciones administrativas que correspondan, se podrán enfrentar a un proceso penal (The United States Department of Justice, 2020).

La autoridad defensora de la competencia en Rusia, en el mes de febrero del año 2019, inició un procedimiento de investigación administrativa por supuestos acuerdos o colusiones entre los productores de mascarillas quirúrgicas, en algunas regiones del país. Uno de los aspectos que la autoridad consideró como extraño, fue el aumento porcentual de manera simultánea en los precios de dichos productos, fue de alrededor de un $150 \%$ hasta un $400 \%$, durante los meses de diciembre del año 2019, enero y febrero del 2020 (Centro de Competencia, 2020).

En este caso, la práctica o supuestas prácticas anticompetitivas se estaban cometiendo por productores de mascarillas quirúrgicas, dichas mascarillas estaban siendo utilizadas por la población para evitar, de acuerdo a las recomendaciones de las autoridades sanitarias, el contagio del SARS-CoV2; por ello, al tratarse de prácticas o presuntas prácticas cometidas 
en productos básicos e indispensables para la población, tuvo especial interés en abrir los expedientes de investigación.

Entre otros ejemplos, en el mes de marzo en Italia, la autoridad de defensa de la competencia, inicio una investigación en contra de las empresas eBay y Amazon, por el aumento excesivo de los precios en el llamado gel antibacterial, ofrecido en sus plataformas, lo anterior, solo a unos días o semanas después de que se hizo público el contagio de COVID-19 en ese país (Centro de Competencia, 2020).

En el mes de marzo, la autoridad de defensa de la competencia en el Reino Unido estableció acciones que incluyeron la investigación minuciosa de mercados que, al parecer, iniciaban con prácticas prohibidas, entre ellas, la fijación de precios por parte de las empresas.

En ese mismo mes, la Competition \& Markets Authority, declara que el SARS-CoV2 es un desafío, no solo en el tema de salud, sino en el tema económico, y que ello puede provocar que las empresas se puedan aprovechar de los consumidores, cobrando precios altos $\mathrm{O}$ haciendo publicidad engañosa.

Es preciso mencionar que a medida que la pandemia empeora y su impacto negativo en la economía se vuelve más serio, la CMA creó un grupo de trabajo para examinar el desarrollo de los mercados, buscando identificar el aumento de precios y los acuerdos que busquen obtener ganancias fuera del proceso de libre competencia.

Uno de los más importantes logros del grupo de trabajo fue proponer que la CMA pueda asesorar al Gobierno del Reino Unido, a que su legislación antimonopolio no interfiera en las medidas que se adopten en beneficio de la salud pública (The United Kingdom Competition \& Market Authority, 2020).

En Sudáfrica se lanzó un mensaje fuerte en el mes de marzo del 2019, se inició la investigación sobre casos de precios elevados, impo- niendo sanciones que van desde el $10 \%$ al $25 \%$ de las ventas anuales de las empresas infractoras, esto por supuesto, se establece como un incentivo para los agentes económicos para no llevar a cabo prácticas de esta naturaleza en perjuicio de los consumidores (Centro de Competencia, 2020).

Por su parte, en el mismo mes de marzo, la autoridad de defensa de la competencia de Brasil, inicio una investigación por precios elevados de manera injustificada, en contra de productores y distribuidores de mascarillas y gel antibacterial, igual que en Italia, a partir del brote del virus en el país (Centro de Competencia, 2020).

La autoridad encargada de la defensa de la competencia en Colombia, realizó un llamado a las empresas con el fin de evitar la comisión de prácticas anticompetitivas, sobre todo, aquellos que van encaminadas a la elevación de precios de manera injustificada, así como todas aquellas qué disminuyan o dañen el proceso de libre competencia y libre concurrencia de los mercados (Centro de Competencia, 2020).

Como se ha expuesto, estar al pendiente de comportamientos anticompetitivos, por parte de las autoridades antimonopolio en tiempos de pandemia, es importante, pero además, dichos comportamientos deberían de ser publicitados de manera más notoria.

Lo anterior, debido a que los ciudadanos deberían saber sobre los agentes económicos que, segados por la ambición de obtener más ganancias, se aprovechan en algo tan sensible e importante para la gente, lo que constituye un acto abusivo, que deja ver los fallos del capital privado frente a las libertades que el Estado le otorga.

Enseguida se presentarán situaciones en las que las autoridades de defensa de la competencia, han sido más tolerantes con el sector empresarial, poniendo como prioridad, el fortalecimiento de la economía. 
Salvar la economía, un objetivo que empata con la prioridad de detener la pandemia Con el avance en la propagación del SARSCoV2, para las autoridades nacionales, ha sido muy difícil la decisión de, por un lado, cuidar a la población de la exposición física en lugares concurridos, situación que implica frenar la actividad económica, el acudir a centros de trabajo, el paro de MIPYMES, entre otras situaciones adversas, y, por otro lado, impulsar la economía.

En México, la COFECE, a través del comunicado COFECE-012-2020, del 27 de marzo de este año, establece su postura sobre la aplicación de la Ley Federal de Competencia Económica ante la actual emergencia sanitaria. En ese sentido y frente a la contingencia ocasionada por la epidemia de COVID-19, considerando que la Secretaría de Salud del Gobierno de la República declaró el inicio de la fase dos, dicha Comisión anuncia que es consciente de las dificultades e inconvenientes que esto genera o puede generar a las empresas mexicanas, y a todos los agentes económicos que participan en la economía nacional, así como a los consumidores $\mathrm{y}$ al buen funcionamiento de los mercados en general.

Con el fin de establecer medidas para que no se interrumpan las cadenas de suministro y la restricción de la oferta de productos, la Comisión estableció que los acuerdos entre competidores no deben ser violatorios de la Ley Federal, y que estos se investigan y en su caso se sancionan, al momento en que las consecuencias sean entre otras, la manipulación de precios, la división o segmentación del mercado, o bien, la restricción en el abasto de bienes y servicios en perjuicio del consumidor.

Por lo anterior, los acuerdos o pactos de colaboración entre agentes económicos, durante el tiempo de contingencia ocasionada por el Covid-19, que sean necesarios para mantener o incrementar la oferta de bienes y servicios, así como para satisfacer la demanda de los mismos, o bien, proteger las cadenas de suministro, evitar la escasez o el acaparamien- to de mercancías, y que siempre y cuando dichos acuerdos no tengan por objeto o efecto el desplazamiento de otros agentes económicos competidores que estén participando en el mercado relevante, se considerarán como apegados a la ley y por lo tanto, no serán objeto de investigación o persecución por parte de la autoridad.

Sin embargo, la Comisión realiza un llamado a los agentes económicos para que no aumenten los precios de sus productos o servicios, a menos de que dichos aumentos estén justificados. Advierte la autoridad que los acuerdos entre competidores que tengan como consecuencia la manipulación de precios, la restricción en el abasto de bienes o servicios, la segmentación de los mercados, así como acordar posturas en licitaciones o abstenerse en ellas, seguirá y en su caso se sancionará de acuerdo con la normatividad antimonopolio del país.

Establece también a través de este comunicado qué, está pendiente del comportamiento de todos los agentes económicos que participen en los llamados mercados sensibles o de primera necesidad de los consumidores, sobre todo en los casos de aumento de precios (COFECE-012-2020).

Esta declaración de la COFECE es un mensaje claro de apoyo y entendimiento del sector empresarial, cuidando en todo momento, los límites de la permisibilidad de las prácticas o acuerdos entre competidores que no tengan como efecto el perjuicio de los mercados.

Adicionalmente, la Comisión, por medio del comunicado COFECE-038-2020, presentó propuestas en materia de competencia para contribuir a la reactivación de la economía mexicana, está compuesta de 12 acciones que van dirigidas al fomento para la participación de las empresas en los mercados que son relevantes para la economía de nuestro país, sobre todo aquellos mercados que tienen impacto en el bolsillo de las familias mexicanas. 
La autoridad reconoce que la emergencia sanitaria ha traído como consecuencia una caída en la producción, en donde las empresas más afectadas son las pequeñas y medianas, por otro lado, las empresas con mayor capital cuentan con mayor probabilidad de permanecer en los mercados.

\section{Las acciones que propone la Comisión son las siguientes:}

1. Fomentar el acceso a créditos a una mayor cantidad de mexicanos, así como a pequeñas empresas, creando un buró público que integre los datos del cumplimiento de pagos en servicios públicos como el agua, la luz, créditos que hayan obtenido de Infonavit o Fovissste;

2. Aumentar las opciones de compra de medicinas a precios más accesibles para las familias, impulsando la entrada al mercado de medicamentos genéricos;

3. Buscar mejorar los costos de la electricidad para que sean más competitivos y que se pueda cumplir con el acceso no discriminatorio a las redes de transmisión y distribución;

4. fomentar la bajada de precios en las gasolinas, mediante el permiso para la importación y expendio de gasolina y diésel, buscando que la cadena de valor tenga más competencia, ellos se puede lograr eliminando requisitos que limitan los permisos de importación;

5. En materia de compras públicas, el gobierno contrate bienes y servicios en las mejores condiciones de calidad y precio, mediante una reforma a la ley de adquisiciones buscando en todo momento el aumento de la libre concurrencia y la libre competencia en los procedimientos de compra;

6. Incorporar la disminución de precios el autotransporte foráneo de pasajeros, realizando una modificación a la regulación, que tenga como fin el aumento de la competencia entre las empresas y que permita, además, la participación de nuevos competidores;

7. En los mercados de gasolinas, gas LP y transporte de carga, buscar la incorporación de más empresas a nivel estatal mediante la emisión de directrices del Consejo Nacional de Mejora Regulatoria;

8. Fomentar lacompra en el exterior de productos que sean requeridos por los consumidores, bajando las medidas restrictivas que existen y que juegan en contra de la población;

9. En el caso de servicios como transporte de pasajeros, puertos y transporte ferroviario, que estén concesionados, y que dichas concesiones están por vencer, llevar a cabo su otorgamiento a través de licitaciones transparentes para que los resultados sean más eficientes para los consumidores;

10. El incremento de la entrada de mercancías por vía marítima, con la propuesta de reformar la ley de puertos, incorporándole principios de competencia económica;

11. Una red articulada el transporte ferroviario para que los productos lleguen a un menor costo a los centros de consumo; $y$,

12. Garantizar que las normas que regulan el mercado no obstaculicen el proceso de libre competencia, proponiendo una reforma constitucional al artículo 105, para que la Comisión pueda presentar acciones de inconstitucionalidad sobre normas generales que violenten los principios de competencia (COFECE-030-2020).

En ese sentido, en Noruega el gobierno concedió recientemente al sector del transporte una excepción temporal por 3 meses, en cuanto a la prohibición de acuerdos o prácticas anticompetitivas, señaladas en la ley de competencia de aquel país. El objetivo del Gobierno con esta medida, fue mantener el transporte de pasajeros y mercancías en Noruega para garantizar el servicio a la población y que, la crisis económica o la desaceleración de la economía, no provoque la suspensión de estos servicios esenciales para los ciudadanos (Ministerio de Industria y Comercio de Noruega, 2020).

La Ministra de Comercio e Industria de Noruega, en el comunicado de fecha 18 de marzo de 2020, mencionó que el Gobierno hará lo que sea necesario para garantizar el acceso de los servicios y bienes que la población requiera, entre ellas, la industria del transporte aéreo, por tierra y por mar, que estará exenta del cumplimiento de la ley durante los 3 meses anun- 
ciados. Este anuncio es muy importante, debido a que la autoridad establece excepciones en el cumplimiento de la ley de competencia, buscando no ser rígidos sino flexibles con las empresas que ofrecen esos servicios (Ministerio de Industria y Comercio de Noruega, 2020).

Imagine que hay 2 empresas que ofrecen los servicios de transporte aéreo de una ciudad a otra, pero con motivo de la pandemia, la demanda ha bajado, y por lo tanto, resulta perjudicial para las 2 empresas seguir ofreciendo el servicio diariamente de manera simultánea, esta situación terminará por parar el servicio de ambas empresas, pero si se les permite llegar a un acuerdo para no competir temporalmente y ofrecer sus servicios un día cada empresa, esta práctica anticompetitiva podría traer como consecuencia la continuidad del servicio de transporte entre esas ciudades

De lo anterior se puede decir que la competencia económica no es el fin sino el vehículo para alcanzar el fin, entonces se preguntaría cuál es el fin, la respuesta encuentra diversos caminos. Existe el escenario de competencia que implica que la población tenga bienes y servicios de mejor calidad a menores precios, pero para contar con eso, no se debe olvidar que lo básico es tener acceso a esos bienes o servicios, por ello, primero hay que contar con los bienes y servicios y después, que sean a mejores precios y de mayor calidad. Por lo comentado, se puede entender un anuncio como el de la Autoridad de Competencia de Noruega.

Otro ejemplo de exenciones del cumplimiento de las leyes de competencia, se tiene en Australia, donde la Australian Competition \& Consumer Commission, permitió que los mayoristas de medicamentos pudieran acordar para facilitar la distribución de medicamentos esenciales, una vez que la autoridad les otorgue el permiso para ello.

Mencionó el presidente de la ACCC que permitir a los mayoristas de las farmacéuticas que acuerden y trabajen juntos, para que los medicamentos puedan ser accesibles para to- da la población durante el tiempo de pandemia, es claramente de interés nacional (Australian Competition \& Consumer Commission, 2020).

\section{CONCLUSIONES}

De lo comentado en el presente trabajo se puede concluir que el SARS-Cov2, ha modificado las posturas en materia de defensa de la competencia de las autoridades antitrust: por un lado, cuidando el buen desarrollo de la economía y sus mercados, por otro, permitiendo tener excepciones a la regla que permitan que los consumidores cuenten con bienes y servicios necesarios.

Esto pone en relieve la idea de que la competencia económica no es el fin, sino un vehículo que sirve para poder alcanzarlo; que los consumidores cuenten con bienes y servicios es prioritario antes de que lo hagan en condiciones de libre competencia. Qué será más importante, contar con alimento en condiciones de competencia imperfecta o no contar con él. Este comentario no implica una manera de desacreditar o minimizar los beneficios y la importancia de la libre competencia y la libre concurrencia, pero en situaciones como las que se están experimentando, se puede ser flexible y priorizar las necesidades.

Por lo que se concluye que en los sistemas de mercado, es importante mantener la competencia sana entre los agentes económicos, sin embargo, en situaciones como la provocada por el SARS-CoV2, lo urgente está primero que lo importante, y los gobiernos tienen que entenderlo, nunca los medios son primero que los fines.

\section{Referencias}

Alemanno, A., (2020) Taming COVID-19 by regulation: An Opportunity for Self-Reflection. Revista Europea de Regulación de Riesgos, V 11, 2, jun 2020, Pp. 187-194.

Australian Competition \& Consumer Commission. (2020). Medicine wholesalers to co-operate on access to pharmaceutical products. https://www.accc.gov.au/ media-release/medicine-wholesalers-to-co-operate-on-access-to-pharmaceutical-products 
Baldwin, R., Weder Di Mauro, B. (2020) "Introduction", Mitigating the COVID Economic Crisis: Act Fast and Do Whatever It Takes 2020. VoxEU.org, Center for Economic Policy Research (CEPR) 18 de marzo 2020. Pp. 18. https://voxeu.org/system/files/epublication/ COVIDEconomicCrisis.pdf

Becker, G. (1998). "Let the marketplace Judge Microsoft”. Business Week (Insdustrial/Technology). New York: april 6, Iss.3572. 26 Pp.

Centro de Competencia. (2020) "Reacciones Comparadas de Agencias de Competencia a raiz de la crisis del Coronavirus" 27/03/2020. https://centrocompetencia.com/reacciones-comparadas-de-agencias-de-competencia-a-raiz-de-la-crisis-del-coronavirus/

Comisión Federal de Competencia Económica de México. (2020). COFECE-012-2020 Postura de la COFECE en términos de la aplicación de la Ley Federal de Competencia Económica ante la emergencia sanitaria. https://www.cofece.mx/postura-cofece-ante-emergencia-sanitaria/

Comisión Federal de Competencia Económica de México. (2020). COFECE-031-2020. Multa COFECE a empresas y personas físicas por coludirse en licitaciones de servicios integrales de laboratorio y banco de sangre que convoca el IMSS e ISSSTE. https://www. cofece.mx/multa-cofece-a-empresas-por-coludirse-en-licitaciones-de-servicios-de-banco-de-sangre/

Comisión Federal de Competencia Económica de México.(2020). COFECE-030-2020.Investiga COFE$C E$ posibles prácticas monopólicas relativas en el mercado de la producción, distribución y comercialización de oxígeno medicinal en el territorio nacional. https:// www.cofece.mx/investiga-cofece-posibles-practicas-monopolicas-relativas-en-mercado-de-oxigeno-medicinal/

Comisión Federal de Competencia Económica de México. (2020). COFECE-030-2020. COFECE propone 12 medidas en materia de competencia econóica para apoyar a la reactivación de la economía mexicana. https://www.cofece.mx/propuestas-en-materia-decompetencia-economica-para-contribuir-a-la-reactivacion-de-la-economia-mexicana/

Comisión Europea. (2020). 2215 DO. Marco temporal relativo a las medidas de ayuda estatal destinadas a respaldar la economía en el cotexto del actual brote de COVID-19. https://ec.europa.eu/competition/ state_aid/what_is_new/TF_consolidated_version_ as_amended_3_april_2020_es.pdf

Competition Bureau Canada. (2020). Statement from the Commissioner of Competition regarding enforcement during the COVID-19 coronavirus situation. https://www.canada.ca/en/competition-bureau/ news/2020/03/statement-from-the-commissioner-of-competition-regarding-enforcement-during-the-covid-19-coronavirus-situation.html
Cooter, R. y Ulen. T. (1998). Derecho y Economía, trad. de Eduardo L. Suárez. México: Fondo de Cultura Económica. 61-63 Pp.

Fernández, O. (2000). La competencia, Madrid: Alianza. 103-104 Pp.

Global Competition Review. 2019. Coronavirus roundup cooperate to feed the nation. https:// globalcompetitionreview.com/article/1216448/ coronavirus-roundup-cooperate-to-\%E2\%80\%9Cfeed-the-nation \% E2\% $80 \% 9 \mathrm{D}-\mathrm{uk}$-government-tells-retailers

Irrazabal, F. (2020) La libre competencia y la peste. Newsletter, https://centrocompetencia.com/la-libre-competencia-y-la-peste/

Ministerio de Industria y Comercio de Noruega. (2020). Las aerolineas tienen el visto bueno para cooperar. https://www.regjeringen.no/no/aktuelt/flyselskapene-gis-klarsignal-til-a-samarbeide/id2693957/

Pascual y Vicente, J. (2000). "La defensa de la competencia: un objetivo político de hoy". Gaceta juridica de la Unión Europea. Madrid: mayo - junio, (207). 9 Pp.

Pérez, C. et al (2008). "La competencia económica y la defensa de la competencia". Revista Jurídica Ars Inveniendi. México: enero - junio, año 4, (6). 49-64 Pp.

Pereznieto. L. y Guerrero. R. (2002). Derecho de la competencia económica. México: Oxford University Press. 150 Pp.

Peña, R. (2020) "Libre competencia y COVID-19". Opinión Técnica 5/marzo 2020, https://librecompetencia.uc.cl/images/Investigacion/Antitrust_Lab/ Opiniones/CO_PLCUC_RP_Marzo_2020.pdf

Petros, A. (2020). "As Its Antitrust Critics Grow Louder, Google Dials Back One of Its Most Controversial Policies". Competition Plicy international CPI Blogs dec/2020. https://www.competitionpolicyinternational.com/amp-it-up-as-its-antitrust-criticsgrow-louder-google-dials-back-one-of-its-most-controversial-policies/

Samuelson, P. (1999). Economía, 16. ed., trad. de Esther Rabasco y Luis Toharia. Madrid: McGraw-Hill. 176-177 Pp.

Samuelson, P. (1999). Economía, 16. ed., trad. de Esther Rabasco y Luis Toharia. Madrid: McGraw-Hill. 176-177 Pp.

Secretaría de Salud, Gobierno de la República de los Estados Unidos Mexicanos, 2020. Lineamiento para la estimación de riesgos del semáforo por regiones COVID-19. Versión 5.1. https://coronavirus.gob.mx/ wp-content/uploads/2020/10/SemaforoCOVID_ Metodo.pdf

Shapiro, C. y Varian. H. (1999). El dominio de la información, trad. de Marina Fominaya. Barcelona: Antoni Bosch. Pp. 294-296.

The United States Department of Justice. (2020). Justice Department Cautions Business Community against violating antitrust laws in the manufacturing, distribution, and sale of public 
health products. https://www.justice.gov/opa/pr/ justice-department-cautions-business-community-against-violating-antitrust-laws-manufacturing?fbclid=IwAR0WtnAzwIESzIQ9_eX5hhaj2YgTtj0AZLXrIzCMoNCbA0X9PB4BJmPQBEY

The United Kingdom Competition \& Market Authority. (2020). The CMA is today launching a taskforce to tackle negative impacts within its remit of the coronavirus (COVID-19) pandemic. https://www.gov. uk/government/news/cma-launches-covid-19-taskforce
Thomas, C. et al. (2020). "Covid-19 and Competition Law-Companies Must Not Quarentine Competition law Compliance". Compettition Policy International. https://www.competitionpolicyinternational.com/covid-19-and-competition-law-companies-must-not-quarantine-competition-law-compliance/ 